\title{
Holographic Renormalization in Dense Medium
}

\author{
Chanyong Park \\ Institute for the Early Universe, Ewha Womans University, Daehyun 11-1, Seoul 120-750, Republic of Korea \\ Correspondence should be addressed to Chanyong Park; cyong21@gmail.com
}

Received 26 May 2014; Revised 15 August 2014; Accepted 21 August 2014; Published 2 September 2014

Academic Editor: Kadayam S. Viswanathan

Copyright (C) 2014 Chanyong Park. This is an open access article distributed under the Creative Commons Attribution License, which permits unrestricted use, distribution, and reproduction in any medium, provided the original work is properly cited. The publication of this article was funded by SCOAP

The holographic renormalization of a charged black brane with or without a dilaton field, whose dual field theory describes a dense medium at finite temperature, is investigated in this paper. In a dense medium, two different thermodynamic descriptions are possible due to an additional conserved charge. These two different thermodynamic ensembles are classified by the asymptotic boundary condition of the bulk gauge field. It is also shown that in the holographic renormalization regularity of all bulk fields can reproduce consistent thermodynamic quantities and that the Bekenstein-Hawking entropy is nothing but the renormalized thermal entropy of the dual field theory. Furthermore, we find that the Reissner-Nordström AdS black brane is dual to a theory with conformal matter as expected, whereas a charged black brane with a nontrivial dilaton profile is mapped to a theory with nonconformal matter although its leading asymptotic geometry still remains as AdS space.

\section{Introduction}

The AdS/CFT correspondence [1-5] is a fascinating tool in studying the strongly interacting system like quantum chromodynamics (QCD) or condensed matter system. Although there is no direct proof for the AdS/CFT correspondence, it is conceptually supported from the same symmetry between a $d+2$-dimensional Anti de Sitter (AdS) gravity and $d+1$ dimensional conformal field theory (CFT). The key relation for this duality is that the semiclassical partition function of AdS gravity corresponds to the generating functional of a dual CFT [1]:

$$
e^{-S_{\text {grav }}}=\left\langle e^{-S}\right\rangle_{\mathrm{CFT}} \text {. }
$$

This relation has been widely used and checked in various works related to integrability and localization [6-8]. For more realistic systems, it is important to encode the matter effect properly which has been considered by considering the dual geometry in [9-11]. In this paper, we will investigate such a matter theory by using the holographic renormalization technique [12-22].

In general, the partition function of a quantum field theory suffers from the UV divergence which should be renormalized by adding appropriate counter terms. This is also true in the dual gravity where the corresponding IR divergence is caused due to infinite volume of the background geometry. In $[12,13]$, it was shown that adding local counter terms to the AdS boundary makes the on-shell gravity action finite and that quantities derived from it can be reinterpreted as those of the dual CFT. This holographic renormalization has been generalized even to the non-AdS geometries like the Lifshitz geometry [23-28] and Einstein-dilaton gravity [2939]. In these non-AdS examples, the renormalized gravity action, after assuming the gauge/gravity duality, was used to understand thermodynamics and hydrodynamics of the dual nonrelativisitic or nonconformal theory. In this procedure, the counter terms can not only get rid of the UV divergence but also yield additional finite contributions, so finding the correct counter terms is crucial for obtaining correct physical quantities. This is also true in the renormalization of a quantum field theory.

In holography, a conformal field theory matches to asymptotic AdS geometry. Introducing matter may break the conformal symmetry depending on the properties of matter. In the strong coupling regime, it is hard to investigate such matter effects through the traditional quantum field theory method. Although many things still remain to be clarified, recent numerous works based on the AdS/CFT 
correspondence provided some clues for understanding a strongly interacting system. In the holographic set-up, matter of the dual CFT can be realized as a field in AdS space. In particular, a massless vector field in AdS is mapped to quark or hadronic number operator in the deconfining or confining phase, respectively [9-11, 40,41]. Note that the black brane geometry is dual to a deconfining phase, while the nonblack brane geometries, thermal AdS, and thermal charged AdS in the hard wall model describe a confining phase. From now on, we concentrate on a charged black brane geometry. 0

The holographic renormalization of the $d+2$-dimensional gravity theory leads to the stress tensor of the $d+1$ dimensional boundary field theory. For a charged black brane, the dual theory contains quark matter. In the thermodynamic point of view, the existence of matter makes two different thermodynamic interpretations possible [42, 43]. On the gravity side, these two different ensembles can be represented by the asymptotic boundary condition of the vector field. For example, the grand canonical ensemble described by the chemical potential corresponds to the Dirichlet boundary condition, while the Neumann boundary condition is dual to the canonical ensemble. These two thermodynamic systems are connected to each other through the Legendre transformation. In a charged black brane, there exists a natural boundary condition for a vector field at the horizon. The regularity of a vector field at the horizon is related to the Legendre transformation. As mentioned before, the holographic renormalization requires appropriate counter therms. In addition, in the canonical ensemble, one more boundary term, the so-called Neumannizing term, is needed to describe the Neumann boundary condition which is crucial to obtain the correct thermodynamics. We show that physical quantities derived by the holographic renormalization perfectly satisfy the thermodynamic law in both thermodynamic ensembles. Intriguingly, in the extremal limit of a Reissner-Nordström AdS (RNAdS) black brane we find that the Fermi surface energy of the dual field theory is proportional to $N^{1 / 3}$ in the UV limit, where $N$ denotes the number of quark. If there exists asymptotic freedom in the dual CFT, one can identify the matter with the free relativistic fermion because it gives rise to $\epsilon_{F} \sim N^{1 / 3}$. However, since there is no asymptotic freedom in the holographic dual CFT, one can not directly interpret it as free relativistic fermions. We also consider another charged black brane in the EinsteinMaxwell-dilaton theory in which the holographic renormalization also leads to the consistent physical quantities satisfying the thermodynamic law. Although the extremal limit is not well defined in this case, one can still think of the Fermi surface in the low temperature limit. The Fermi surface energy of the Einstein-Maxwell-dilaton theory is also proportional to $\epsilon_{F} \sim N^{1 / 3}$ similar to the RNAdS black brane case. The holographic renormalization results show that dual matter of the RNAdS black brane does not break the conformal symmetry at the UV fixed point, whereas dual matter of the Einstein-Maxwell-dilaton theory breaks the conformal symmetry in spite of the asymptotic AdS geometry.
The rest of paper is organized as follows. In Section 2, after applying the holographic renormalization to the EinsteinMaxwell theory, we calculate the on-shell actions and boundary stress tensors of two thermodynamic ensembles. From these result, we derive various thermodynamic quantities and show that they satisfy the thermodynamic law. In Section 3, the holographic renormalization technique is applied to another asymptotic charged AdS black brane of the EinsteinMaxwell-dilaton theory. Finally, we finish this work with some concluding remarks.

\section{Holographic Renormalization of the RNAdS Black Brane}

Let us first take into account a 5-dimensional RNAdS black brane. A charged black brane solution usually has two hairs, charge, and mass, so that its thermodynamics can be represented as two different thermodynamic systems depending on the choice of the fundamental thermodynamic variables [42]. On the gravity theory side, the choice of ensemble is closely related to the asymptotic boundary condition of a bulk gauge field. For example, imposing the Dirichlet boundary condition on the gauge field corresponds to taking a grand canonical ensemble, whereas one should impose the Neumann boundary condition in order to describe a canonical ensemble. In general, those two thermodynamic systems are related to each other by the Legendre transformation (see the details in the Appendix). According to the AdS/CFT correspondence, the black brane thermodynamics can be identified with that of the dual field theory $[5,13,25-27$, $30,31,38,43]$. In this section, by using the holographic renormalization we will investigate thermodynamics of the field theory dual to the RNAdS black brane. As will be shown, the holographic renormalization results are perfectly matched to the black brane thermodynamics.

For the direct comparison with the black brane thermodynamics, we regard an Euclidean Einstein-Maxwell action with a negative cosmological constant. To obtain the Euclidean action from the Lorentzian one in (A.1), one can apply Wick rotation which describes rotation of time coordinate in a complex plane:

$$
x^{t} \longrightarrow-i x^{\tau}, \quad x_{t} \longrightarrow i x_{\tau} .
$$

Under this Wick rotation, the field strength $F_{r t}$ with a Lorentzian signature transforms to

$$
F_{r t} \longrightarrow \partial_{r} A_{t}-i \partial_{\tau} A_{r}
$$

In order to preserve the usual definition form of the field strength $F_{M N}=\partial_{M} A_{N}-\partial_{N} A_{M}$ up to an overall factor, we should also rotate the time component of the gauge field as $A_{t} \rightarrow i A_{\tau}$. Then, the Lorentzian field strength changes to the Euclidean one, $i F_{r \tau}$, with $A_{M}=\left\{A_{\tau}, \vec{A}\right\}$. These Wick rotations lead to the following Euclidean action:

$$
S_{E}=\int d^{5} x \sqrt{g}\left[-\frac{1}{2 \kappa^{2}}(\mathscr{R}-2 \Lambda)+\frac{1}{4 g^{2}} F_{M N} F^{M N}\right],
$$

where the Euclidean metric is used. Since the Euclidean action is the same as the Lorentzian one up to an overall 
minus sign, all equations of motion are the same only except the fact that the Euclidean metric should be used. For example, the Euclidean Einstein and Maxwell equations have the same forms as the Lorentzian ones in (A.2), which allows the following Euclidean RNAdS black brane solution [9]:

$$
\begin{aligned}
& A_{\tau}=-i A_{t}=-i\left(g^{2} \mu-\frac{Q}{2 r^{2}}\right), \\
& d s^{2}=r^{2} f(r) d \tau^{2}+\frac{d r^{2}}{r^{2} f(r)}+r^{2}\left(d x^{2}+d y^{2}+d z^{2}\right),
\end{aligned}
$$

with

$$
f(r)=1-\frac{m}{r^{4}}+\frac{\kappa^{2}}{6 g^{2}} \frac{Q^{2}}{r^{6}},
$$

where $m$ and $\mu$ are a black brane mass and chemical potential, respectively, and we define its electric charge as $\sqrt{\left(\kappa^{2} / 6 g^{2}\right)} Q$ for later convenience. As mentioned before, a charged black brane has two thermodynamic interpretations due to the conserved charge. In the thermodynamic point of view, one corresponds to the grand canonical ensemble described by temperature, volume, and chemical potential. If one uses the charge as a fundamental variable instead of the chemical potential, the black brane thermodynamics must be represented as the canonical ensemble.

The requirement of the regular metric at the horizon generally leads to specific Euclidean time periodicity $\beta$ corresponding to the inverse Hawking temperature. Due to the existence of the well-defined temperature, one can expect that the black brane may be regarded as a thermal system. This is true if the entropy is given by the Bekenstein-Hawking entropy proportional to the horizon area. This thermodynamic interpretation causes a big issue, why the entropy is proportional to the area but not to volume. There have been many attempts to account for this area law. One of them is the holographic principle in which people have tried to map the black brane thermodynamics to that of the boundary theory. Recently, the concept of the holography has been further developed to the gauge/gravity duality of the string theory and becomes a fascinating tool in studying a strongly interacting system $[1-5,20]$. Following this holographic interpretation the area related to the Bekenstein-Hawking entropy is identified with the volume of the boundary theory, where the Bekenstein-Hawking entropy behaves as an extensive quantity expected in ordinary thermodynamics. However, even in this case it is still mysterious why the Bekenstein-Hawking entropy is defined at the horizon not the boundary. In general, the Hawking temperature is interpreted as temperature detected by an observer living at the asymptotic boundary. Similarly other conserved quantities are also defined at the asymptotic boundary. In order to get welldefined thermodynamics of the boundary theory, it must be understood why the Bekenstein-Hawking entropy appears as the thermal entropy of the dual theory. The holographic renormalization together with natural assumptions, as will be shown, indicates that the renormalized thermal entropy of the boundary theory is exactly given by the BekensteinHawking entropy.
Physically, it is quite natural to assume that all fields are regular in the entire space. If not so, the theory becomes singular. In the Einstein-Maxwell theory, there exist two bulk fields, metric and vector fields. At the horizon, the absence of the conical singularity in the metric determines the time periodicity. The Hawking temperature is represented as the inverse of the time periodicity:

$$
T_{H}=\frac{r_{h}}{\pi}-\frac{\kappa^{2} Q^{2}}{12 \pi g^{2}} \frac{1}{r_{h}^{5}},
$$

where the horizon $r_{h}$ satisfies $f\left(r_{h}\right)=0$. Although the Hawking temperature is evaluated at the horizon, as mentioned before, it should be regarded as temperature at the asymptotic boundary. Under the coordinate transformation, the invariant combination of the vector field is given by $g^{\mu \nu} A_{\mu} A_{\nu}$ which reduces to $g^{\tau \tau} A_{\tau} A_{\tau}$ on the RNAdS black brane geometry. Since $g^{\tau \tau}$ diverges at the horizon, the regularity requires that $A_{\tau}$ should vanish at the horizon. This fact yields a relation between $\mu$ and $Q$ :

$$
\mu=\frac{Q}{2 g^{2} r_{h}^{2}}
$$

In the holographic renormalization, these two consequences of natural assumptions can derive all desired thermodynamic quantities as will be shown. It is worth to note that two parameters of the black brane, $m$ and $Q$, can be represented as functions of $T_{H}$ and $\mu$ (or $Q$ depending on the boundary condition of the vector field) [9]. From now on, we take into account $T_{H}$ and $\mu$ (or $Q$ ) as fundamental parameters in which $r_{h}$ becomes a function of them.

In general, the gravity action without additional boundary terms is not well defined because its variation becomes problematic at the boundary. Therefore, the following two boundary terms should be added:

$$
S_{b}=\frac{1}{\kappa^{2}} \int_{\partial \mathscr{M}} d^{d} x \sqrt{\gamma} \Theta+\frac{\zeta}{g^{2}} \int_{\partial \mathscr{M}} d^{4} x \sqrt{\gamma} n^{r} F_{r \tau} A_{\tau},
$$

where $\gamma_{a b}$ and $\Theta$ denote the induced metric and the extrinsic curvature. The unit normal vector is given by $n^{r}=\sqrt{g^{r r}}$. The first is the Gibbons-Hawking term and the last is called the Neumannizing term which determines the boundary condition of $A_{\tau}$, for example, $\zeta=0$ or 1 for the Dirichlet or Neumann boundary condition, respectively [23]. As a consequence, the action having the well defined variations is given by the sum of the bulk in (4) and boundary actions in (9).

The useful and crucial relation in the AdS/CFT correspondence is that the on-shell gravity action is proportional to the free energy (or the generating functional) of the boundary (or dual) field theory:

$$
e^{-S_{\mathrm{on}}}=\left\langle e^{-S}\right\rangle_{\mathrm{CFT}}=e^{-\beta F}
$$

where $F$ means the boundary free energy [1]. Despite the previous regularity assumption at the asymptotic boundary, the on-shell gravity action still suffers from some divergences 
caused by infinity in $r$. Following the holographic principle, these divergences can be reinterpreted as the UV divergences of the dual field theory and removed by appropriate local counter terms analogous to quantum field theory. This method is called the holographic renormalization [13]. In this procedure counter terms not only remove the UV divergences but also provide finite contributions. Since physics crucially depends on these finite contributions finding the correct counter terms is really an important issue.

The correct counter terms should satisfy the following prescriptions.

(i) The on-shell action should be finite.

(ii) The variation of the on-shell action with respect to the leading boundary value of all bulk fields should also be finite.

As mentioned before, the first prescription corresponds to finiteness of the free energy. The second implies the physical quantities of the dual theory should be also finite. In order to understand this, it should be noticed that, in general, the bulk fields denoted by $\Phi$ collectively have the following expansion form at the asymptotic boundary [2-4]:

$$
\Phi=J r^{m_{+}}(1+\cdots)+\langle\overline{\mathcal{O}}\rangle r^{m_{-}}(1+\cdots),
$$

where ellipsis denotes small corrections. If $m_{+}>m_{-}$, on the dual field theory side, $J$ and $\overline{\mathcal{O}}$ correspond to a source and vev of its dual density operator, respectively and $\overline{\mathcal{O}}$ becomes a conjugate variable of $J$. The on-shell gravity action, $S_{\text {on }}$, then includes the term related to the generating functional:

$$
S_{\text {on }}=\cdots+\int d \tau \int d^{3} x J\langle\overline{\mathcal{O}}\rangle+\cdots
$$

where the Euclidean time integral runs from 0 to $\beta$. Defining $\langle\mathcal{O}\rangle=\int d^{3} x\langle\overline{\mathcal{O}}\rangle$, the one point correlation function, when the source $J$ is uniform, is given by

$$
\beta\langle\mathcal{O}\rangle=-\frac{\partial S_{\mathrm{on}}}{\partial J},
$$

where the inverse temperature, $\beta$, comes from the Euclidean time integral. Therefore, the second prescription implies the one point function obtained by the holographic renormalization should be finite:

$$
\langle\mathcal{O}\rangle=-\frac{1}{\beta} \frac{\partial S_{\mathrm{on}}}{\partial J}=\text { finite. }
$$

It is consistent with our intuition. Since $\langle\mathcal{O}\rangle$ is a physical observable, it must be finite. In the Einstein-Maxwell theory there are two bulk fields, so the second prescription leads to

$$
\left\langle T_{a b}\right\rangle=-\frac{2}{\beta} \frac{\partial S_{\text {on }}}{\partial \gamma^{a b}}, \quad\langle N\rangle=-\frac{1}{\beta} \frac{\partial S_{\text {on }}}{\partial \mu},
$$

where the factor 2 is introduced for later convenience. In the AdS/CFT correspondence, the electric charge of the RNAdS black brane is related to the quark number density operator of the dual field theory. So the quark number operator becomes $N=Q V_{3}$, where $V_{3}$ is the regularized volume at the asymptotic boundary.
2.1. Grand Canonical Ensemble at the UV Fixed Point. In the charged black brane geometry, the regularity conditions at the horizon in (7) and (8) determine two integration constants of the Einstein and Maxwell equations. After requiring the asymptotic AdS geometry, however, there still remains one integration constant in the vector field which should be fixed by another boundary condition. One can usually choose a Dirichlet or Neumann boundary condition at the asymptotic boundary.

Let us first impose the Dirichlet boundary condition on the gauge field, which fixes the chemical potential corresponding to the boundary value of the gauge field. Then, the dual theory is described by the grand potential of a grand canonical ensemble. In this case, since the variation of the renormalized action with respect to the gauge field is well defined, an additional boundary term is not needed. When the Neumann boundary condition instead of the Dirichlet boundary condition is imposed, this is not true anymore as will be shown in the next section. The resulting renormalized action with the Dirichlet boundary condition becomes

$$
S_{\text {ren }}^{\text {grand }}=S_{E}+S_{\mathrm{GH}}+S_{\mathrm{ct}} \text {, }
$$

where $S_{\mathrm{ct}}$ means the counter terms. For the asymptotic AdS space, the correct counter terms have been found in [13]. In the Poincare patch of a five-dimensional AdS space, the counter term is given by

$$
S_{\mathrm{ct}}=\frac{3}{\kappa^{2}} \int_{\partial \mathscr{M}} d^{4} x \sqrt{\gamma}
$$

In the RNAdS black brane the vector field depending only on the radial coordinate does not generate a new divergence, so an additional counter term is not required.

From the renormalized action, one can easily read the thermodynamic quantities by evaluating the boundary stress tensor. At the UV fixed point $\left(r_{0} \rightarrow \infty\right)$, the grand potential from the on-shell gravity action yields

$$
\begin{aligned}
\Omega & \equiv \lim _{r_{0} \rightarrow \infty} \frac{S_{\text {ren }}^{\text {grand }}}{\beta}, \\
& =-\frac{V_{3}}{2 \kappa^{2}} r_{h}^{4}-\frac{1}{3} g^{2} \mu^{2} V_{3} r_{h}^{2},
\end{aligned}
$$

where (8) is used. This grand potential is consistent with the result obtained from the black brane thermodynamics (A.21). The renormalized boundary energy-momentum tensor is then defined as [12]

$$
\begin{aligned}
\left\langle T^{\mu}{ }_{\nu}\right\rangle & \equiv \lim _{r_{0} \rightarrow \infty}\left(-\frac{2 \gamma^{\mu \rho}}{\beta} \frac{\delta S_{\mathrm{ren}}^{\mathrm{grand}}}{\delta \gamma^{\rho \nu}}\right) \\
& =\lim _{r_{0} \rightarrow \infty}\left(\frac{1}{\kappa^{2}} \int d^{3} x \sqrt{\gamma}\left(\Theta^{\mu}{ }_{\nu}-\delta^{\mu}{ }_{\nu} \Theta\right)-2 \gamma^{\mu \rho} \frac{\delta S_{\mathrm{ct}}}{\delta \gamma^{\rho \nu}}\right),
\end{aligned}
$$


where the integral implies the boundary spatial volume integral. The explicit internal energy and pressure read

$$
\begin{aligned}
& E=T_{0}^{0}=\frac{3 V_{3}}{2 \kappa^{2}} r_{h}^{4}+g^{2} V_{3} \mu^{2} r_{h}^{2}, \\
& P=-\frac{T_{i}^{i}}{V_{3}}=\frac{1}{2 \kappa^{2}} r_{h}^{4}+\frac{1}{3} g^{2} \mu^{2} r_{h}^{2},
\end{aligned}
$$

where $i$ is not summed. From the previous second prescription the vev of the number operator becomes

$$
\langle N\rangle \equiv \lim _{r_{0} \rightarrow \infty}\left(-\frac{1}{\beta} \frac{\partial S_{\mathrm{ren}}^{\mathrm{grand}}}{\partial \mu}\right)=2 g^{2} V_{3} r_{h}^{2} \mu .
$$

When imposing the Dirichlet boundary condition on the vector field, all physical quantities must be represented as functions of $T_{H}, V_{3}$, and $\mu$ which are fundamental variables describing the dual field theory. Since the grand potential of this system is also a function of those variables, the following identity naturally appears:

$$
d \Omega=\frac{\partial \Omega}{\partial T_{H}} d T_{H}+\frac{\partial \Omega}{\partial \mu} d \mu+\frac{\partial \Omega}{\partial V_{3}} d V_{3} .
$$

Since the grand potential in (18) is linearly proportional to $V_{3}$, its derivative with respect to $V_{3}$ satisfies

$$
\frac{\partial \Omega}{\partial V_{3}}=-P \text {. }
$$

Comparing $\partial \Omega / \partial \mu$ with (21), we can easily see

$$
\frac{\partial \Omega}{\partial \mu}=-N
$$

where $\langle N\rangle$ is denoted by $N$. These relations indicate that the conjugate variables of $V_{3}$ and $\mu$ are given by $P$ and $N$, respectively. Now, let us define

$$
S \equiv-\frac{\partial \Omega}{\partial T_{H}}
$$

and then $S$ becomes the conjugate variable of $T_{H}$. If the system we consider is a thermal system, $S$ can be naturally identified with the thermal entropy. Before checking the first law of thermodynamics, however, we can not say yet that our system is in thermal equilibrium. Using the fact that $r_{h}$ is given by a function of $T_{H}$ and $\mu, S$ at fixed $\mu$ and $V_{3}$ reduces to

$$
S=-\left.\frac{\partial \Omega}{\partial T_{H}}\right|_{\mu, V_{3}}=\frac{2 \pi V_{3}}{\kappa^{2}} r_{h}^{3} .
$$

The values obtained here automatically satisfy the following relation:

$$
\Omega=E-T_{H} S-\mu N,
$$

which together with the exact differential in (22) leads to the first law of thermodynamics

$$
d E=T_{H} d S+\mu d N-P d V_{3}
$$

These results show that the dual field theory is really a thermal system, as one expected. Since the grand potential is the renormalized quantity of the dual field theory, the derived $S$ from it is also the renormalized one. As shown before, it satisfies all thermodynamic relations so that it should be identified with the thermal entropy of the dual field theory. Intriguingly, the thermal entropy derived here is exactly the same as the Bekenstein-Hawking entropy in (A.9). As a result, the holographic renormalization only with the quite natural regularity conditions leads to thermodynamics of the dual theory which is coincident with the black brane thermodynamics. In this case, all thermodynamic quantities have appropriate dual theory interpretations. In particular, the Bekenstein-Hawking entropy appears as the renormalized thermal entropy.

Note that the chemical potential $\mu$ and quark number operator $N=Q V_{3}$ are conjugate variables and, following the AdS/CFT correspondence, $\mu$ is a source of $N$ (or Q) in the dual field theory. Since the grand potential is described by the chemical potential, one can easily evaluate the correlation function of the number operator in the grand canonical ensemble. At the UV fixed point $\left(r_{0} \rightarrow \infty\right)$, the one-point function of the number operator is given by (21), which on the gravity side corresponds to the electric charge of the black brane. One can easily check that this one point function coincides with the regularity of the vector field in (8). Moreover, as will be seen in the next section, it is also related to the Legendre transformation of the thermodynamic system. From (7) and (8), $r_{h}$ can be rewritten in terms of $\mu$ and $T_{H}$ as

$$
r_{h}=\frac{\pi T_{H}+\sqrt{\pi^{2} T_{H}^{2}+(4 / 3) \kappa^{2} g^{2} \mu^{2}}}{2} .
$$

Using this, the expectation value of the number operator can be rewritten, in terms of the fundamental thermodynamic variables of the grand canonical ensemble, as

$$
\langle N\rangle=\frac{g^{2} V^{3}}{2} \mu\left(\pi T_{H}+\sqrt{\pi^{2} T_{H}^{2}+\frac{4}{3} \kappa^{2} g^{2} \mu^{2}}\right)^{2} .
$$

In particular, at zero temperature $\left(r_{h}=\kappa g \mu / \sqrt{3}\right)$ this correlator simply reduces to

$$
\langle N\rangle=\frac{2}{3} g^{4} V_{3} \kappa^{2} \mu^{3} .
$$

Interestingly, this zero temperature expectation value of the number operator shows that the Fermi surface energy $\left(\epsilon_{F}=\right.$ $\mu$ ), if it exists [44], is given by

$$
\epsilon_{F}=\left(\frac{3}{2 \kappa^{2} g^{4}}\right)^{1 / 3}\left(\frac{N}{V_{3}}\right)^{1 / 3},
$$

where $N$ denotes the expectation value of the number operator. In the general statistical mechanics, the Fermi surface energy of a nonrelativistic free fermions, due to the nonrelativistic dispersion relation $\epsilon=p^{2} / 2 m$, yields

$$
\epsilon_{F} \sim\left(\frac{N}{V_{3}}\right)^{2 / 3} \text {. }
$$


On the other hand, for the relativistic free fermions with the dispersion relation, $\epsilon=p$, the Fermi surface energy in the natural unit is given by

$$
\epsilon_{F}=\left(3 \pi^{2}\right)^{1 / 3}\left(\frac{N}{V_{3}}\right)^{1 / 3}
$$

which looks very similar to the dual field theory result in (32). If the holographic dual theory of the asymptotic AdS geometry has asymptotic freedom, one can easily identify the holographic dual matter with the relativistic free fermion. Unfortunately, because of the absence of an asymptotic freedom in the dual theory of the asymptotic AdS space, one can not naively interpret the holographic dual matter as free fermion. So it would be interesting to construct the dual geometry of the field theory with the asymptotic freedom and study it further. Although the dual matter of the RNAdS geometry is not a relativistic free fermion, it shows the relativistic free fermion-like Fermi surface energy which might be related to physical property of the non-Fermi liquid [44].

Before concluding this section, it is worth to note that the trace of the boundary stress tensor vanishes. Since the second terms in (20) are contributions from the holographic matter, this result says that the holographic dual matter of the RNAdS black brane geometry is also conformal. This is not always true in the asymptotic AdS geometry. As will be shown later, a different asymptotic charged AdS black brane solution shows that its dual matter can break the boundary conformal symmetry in spite of the asymptotic AdS geometry.

2.2. Canonical Ensemble. As shown in [9], imposing the Neumann boundary condition instead of the Dirichlet boundary condition corresponds to the Legendre transformation in the dual theory, which describes a canonical ensemble instead of a grand canonical ensemble. Although there is no required additional counter term similar to the grand canonical ensemble case, a new boundary term should be added to satisfy the Neumann boundary condition. Varying the gauge kinetic term with respect to the gauge field leads to a Neumannizing term [23]:

$$
\delta S_{N}=\frac{1}{g^{2}} \int_{\partial \mathscr{M}} d^{4} x \sqrt{g} g^{r r} g^{\tau \tau} F_{r \tau} \delta A_{\tau} .
$$

This term, when the Dirichlet boundary condition is imposed, automatically vanishes due to $\delta A_{\tau}=0$, whereas it does not automatically vanish when the Neumann boundary condition is imposed. In order to get a well defined gauge field variation with the Neumann boundary condition, one should add an additional boundary term

$$
S_{b d}=\frac{1}{g^{2}} \int_{\partial \mathscr{M}} d^{4} x A_{\mu} J^{\mu},
$$

where $A_{\mu}=\left\{A_{\tau}, 0,0,0\right\}$ and $J^{\mu}=\{i Q, 0,0,0\}$. Then, the variation of this new boundary term together with (35) gives rise to

$$
\begin{aligned}
\delta S & =\delta S_{N}+\delta S_{b d}, \\
& =\frac{1}{g^{2}} \int_{\partial \mathscr{M}} d^{4} x\left(\sqrt{g} g^{r r} g^{\tau \tau} F_{r \tau}+i Q\right) \delta A_{\tau} .
\end{aligned}
$$

The vanishing of the action variation leads to the following Neumann boundary condition:

$$
\sqrt{g} g^{r r} g^{\tau \tau} F_{r \tau}=-i Q
$$

which is nothing but the Euclidean version of the Nuemann boundary condition in (A.6). Therefore, the resulting renormalized action for the canonical ensemble becomes

$$
S_{\text {ren }}^{\mathrm{can}}=S_{E}+S_{\mathrm{GH}}+S_{b d}+S_{\mathrm{ct}} \text {. }
$$

From this renormalized action, the free energy of the dual canonical ensemble is given by

$$
\begin{aligned}
F & \equiv \lim _{r_{0} \rightarrow \infty} \frac{S_{\text {ren }}^{\mathrm{can}}}{\beta}, \\
& =-\frac{V_{3}}{2 \kappa^{2}} r_{h}^{4}+\frac{5 N^{2}}{12 g^{2} V_{3}} \frac{1}{r_{h}^{2}},
\end{aligned}
$$

where $r_{h}$ should be regarded as a function of $T_{H}$ and $N$. Comparing the free energy with the grand potential in (18) says that on the dual field theory side the new boundary term reduces to $S_{b d} / \beta=\mu N$ and that the free energy and the grand potential are related by the Legendre transformation as they should do. After evaluating the boundary stress tensor similar to the previous case, one can easily read the internal energy and pressure in terms of $N$ :

$$
\begin{aligned}
E & =T_{0}^{0} \equiv \lim _{r_{0} \rightarrow \infty}\left(-\frac{2 \gamma^{0 \rho}}{\beta} \frac{\delta S_{\text {ren }}^{\mathrm{can}}}{\delta \gamma^{\rho 0}}\right), \\
& =\frac{3 V_{3}}{2 \kappa^{2}} r_{h}^{4}+\frac{N^{2}}{4 g^{2} V_{3}} \frac{1}{r_{h}^{2}}, \\
P_{i} & =-\frac{T_{i}^{i}}{V_{3}} \equiv \lim _{r_{0} \rightarrow \infty}\left(\frac{2 \gamma^{i \rho}}{\beta V_{3}} \frac{\delta S_{\text {ren }}^{\mathrm{can}}}{\delta \gamma^{\rho i}}\right), \\
& =\frac{1}{2 \kappa^{2}} r_{h}^{4}+\frac{N^{2}}{12 g^{2} V_{3}^{2}} \frac{1}{r_{h}^{2}} .
\end{aligned}
$$

These results exactly coincide with the RNAdS black brane thermodynamics of the canonical ensemble in (A.13) and (A.16).

\section{Holographic Nonconformal Matter in the Asymptotic AdS Geometry}

One can also take into account another deformed charged black brane which still has an asymptotic AdS geometry. One of the known examples is the deformed RNAdS black 
brane with two different Liouville scalar potentials, where the scalar field was identified with a dilaton because it controls the physical coupling [45-48]. In general, the dilaton field is related to the running coupling of the dual field theory, so the existence of the nontrivial dilaton field implies that the dual field theory is deviated from the UV conformal fixed point if the dual operator of the dilaton is relevant. In the deformed charged black brane, the dilaton is coupled to the gauge field dual to the matter field so that this nontrivial dilaton coupling can change the property of the dual matter field. By using the the holographic renormalization studied in the previous section, we will investigate the dual field theory of the deformed charged black brane and the property of the dual matter.

With the Lorentzian signature, the action describing the deformed charged black brane is given by

$$
\begin{aligned}
S= & \frac{1}{2 \kappa^{2}} \int d^{5} x \sqrt{-g} \\
& \times\left[\mathscr{R}-\frac{1}{4} e^{4 \phi} F_{\mu \nu} F^{\mu \nu}-12 \partial_{\mu} \phi \partial^{\mu} \phi+\frac{1}{R^{2}}\left(8 e^{2 \phi}+4 e^{-4 \phi}\right)\right],
\end{aligned}
$$

where $R$ denotes the AdS radius. From now on, we set $R=1$ for convenience. Then, the known solutions are

$$
\begin{aligned}
d s^{2} & =r^{2} e^{2 A(r)}\left(-f(r) d t^{2}+d \vec{x}^{2}\right)+\frac{e^{2 B(r)}}{r^{2} f(r)} d r^{2}, \\
A_{\mu} d x^{m} & =A_{t} d t,
\end{aligned}
$$

with

$$
\begin{aligned}
A(r) & =\frac{1}{3} \log \left(1+\frac{Q^{2}}{8 m r^{2}}\right), \\
B(r) & =-\frac{2}{3} \log \left(1+\frac{Q^{2}}{8 m r^{2}}\right), \\
f(r) & =1-\frac{m}{\left(r^{2}+Q^{2} / 8 m\right)^{2}}, \\
A_{t} & =2 \kappa^{2} \mu-\frac{Q}{2\left(r^{2}+Q^{2} / 8 m\right)}, \\
\phi(r) & =\frac{1}{6} \log \left(1+\frac{Q^{2}}{8 m r^{2}}\right),
\end{aligned}
$$

where we used slightly different notations from [45] for more concrete thermodynamic interpretation. In the above, free parameters, $m, Q$, and $\mu$, correspond to the mass, charge, and chemical potential of the charged black brane, respectively. More precisely, if one expands the gauge field near the asymptotic boundary to

$$
A_{t}=2 \kappa^{2} \mu-\frac{Q}{2 r^{2}}+\mathcal{O}\left(\frac{1}{r^{4}}\right)
$$

two integration constants $\mu$ and $Q$ appear. Furthermore, following the AdS/CFT correspondence, these values can be reinterpreted, similar to the previous case, as the quark chemical potential $\mu$ and number density $Q$

$$
Q=\lim _{r \rightarrow 0} \sqrt{-g} e^{4 \phi} g^{t t} g^{r r} \partial_{r} A_{t}
$$

Note that, for $Q \rightarrow 0$, the above solution simply reduces to the usual Schwarzschild AdS black brane.

The absence of a conical singularity at the horizon gives rise to Hawking temperature which is associated with the horizon $r_{h}$ :

$$
r_{h}=\pi T_{H}
$$

and the Bekenstein-Hawking entropy leads to

$$
S_{\mathrm{BH}}=\frac{2 \pi V_{3}}{\kappa^{2}}\left(r_{h}^{3}+\frac{Q^{2}}{8 m} r_{h}\right) \text {. }
$$

From the vanishing of the black brane factor and the gauge potential at the horizon, one can easily find two relations between parameters:

$$
\begin{gathered}
Q=4 \kappa^{2} \mu \sqrt{m}, \\
\sqrt{m}=r_{h}^{2}+2 \kappa^{4} \mu^{2},
\end{gathered}
$$

which are convenient in describing the grand canonical ensemble because all other parameters are given by functions of $T_{H}$ and $\mu$. In the canonical ensemble, $m$ and $\mu$ should be considered as functions of $T_{H}$ and $Q$, which have complicated forms:

$$
\begin{gathered}
\mu=\frac{Q}{4 \kappa^{2} \sqrt{m}}, \\
\sqrt{m}=\frac{1}{3} r_{h}^{2}+\frac{2^{4 / 3} r_{h}^{4}}{3\left(16 r_{h}^{6}+27 Q^{2}+3 Q \sqrt{96 r_{h}^{6}+81 Q^{2}}\right)^{1 / 3}} \\
+\frac{\left(16 r_{h}^{6}+27 Q^{2}+3 Q \sqrt{96 r_{h}^{6}+81 Q^{2}}\right)^{1 / 3}}{32^{4 / 3}} .
\end{gathered}
$$

From the thermodynamic point of view, since $m$ is given by a very complicated function of $T_{H}$ and $Q$ in the canonical ensemble, it is not easy to find analytic thermodynamic quantities directly from the thermodynamic laws. From now on, we try to evaluate the thermodynamic quantities through the holographic renormalization scheme instead of the thermodynamic laws.

With the Euclidean signature, the action for the grand canonical ensemble is

$$
\begin{aligned}
S_{\text {grand }}= & -\frac{1}{2 \kappa^{2}} \int d^{5} x \sqrt{-g} \\
& \times\left[\mathscr{R}-\frac{1}{4} e^{4 \phi} F_{\mu \nu} F^{\mu \nu}-12 \partial_{\mu} \phi \partial^{\mu} \phi\right. \\
& \left.+\frac{1}{R^{2}}\left(8 e^{2 \phi}+4 e^{-4 \phi}\right)\right] .
\end{aligned}
$$


In the holographic renormalization, one should add the Gibbons-Hawking and counter terms in (9). If we impose the Dirichlet boundary condition on $A_{\tau}$, the dual thermodynamics represents the grand canonical ensemble in which the chemical potential instead of the number operator becomes a basic thermodynamic variable. Then, the grand potential proportional to the on-shell gravity action leads at the asymptotic boundary to

$$
\begin{aligned}
\Omega\left(T_{H}, \mu, V_{3}\right) & \equiv \frac{S_{E}}{\beta} \\
& =-\frac{\pi^{4} V_{3}}{2 \kappa^{2}} T_{H}^{4}-2 \pi^{2} \kappa^{2} V_{3} T_{H}^{2} \mu^{2}-\frac{10}{3} \kappa^{6} V_{3} \mu^{4}
\end{aligned}
$$

Furthermore, from the boundary stress tensor, the internal energy and pressure are represented as functions of $T_{H}$ and $\mu:$

$$
\begin{aligned}
E & \equiv T_{0}^{0} \\
& =\frac{3 \pi^{4} V_{3}}{2 \kappa^{2}} T_{H}^{4}+6 \pi^{2} \kappa^{2} V_{3} T_{H}^{2} \mu^{2}+\frac{14}{3} \kappa^{6} V_{3} \mu^{4}, \\
P & \equiv-\frac{T_{i}^{i}}{V_{3}} \\
& =\frac{\pi^{4}}{2 \kappa^{2}} T_{H}^{4}+2 \pi^{2} \kappa^{2} T_{H}^{2} \mu^{2}+\frac{10}{3} \kappa^{6} \mu^{4} .
\end{aligned}
$$

The one-point function of the number operator is

$$
N \equiv \lim _{r_{0} \rightarrow \infty}\left(-\frac{1}{\beta} \frac{\partial S_{\text {ren }}^{\text {grand }}}{\partial \mu}\right)=\frac{4}{3} \kappa^{2} \mu V_{3}\left(3 \pi^{2} T_{H}^{2}+10 \kappa^{4} \mu^{2}\right) .
$$

Similar to the holographic renormalization of the RNAdS black brane, the renormalized thermal entropy $S$ appears as the conjugate variable of $T_{H}$

$$
S \equiv-\left.\frac{\partial \Omega}{\partial T_{H}}\right|_{\mu, V_{3}}=\frac{2 \pi^{2} V_{3} T_{H}}{\kappa^{2}}\left(\pi^{2} T_{H}^{2}+2 \kappa^{4} \mu^{2}\right)
$$

and satisfies the thermodynamic relation

$$
\Omega=E-T_{H} S-\mu N .
$$

Comparing it to the Bekenstein-Hawking entropy in (48), one can easily see that the derived thermal entropy is exactly the same as the Bekenstein-Hawking entropy and all thermodynamic quantities derived here are consistent with those obtained from the black brane geometry.

For $\mu=0$, the thermodynamics of the Schwarzschild AdS black brane is reproduced. When $Q=2^{3 / 2} \mathrm{~m}^{3 / 4}$, the charged black brane reaches the extremal limit, where $T_{H}$ and $S_{B H}$ reduce to zero. It should be however noted that the extremal limit has naked singularity at the center $(r=0)$ so that the zero temperature is not well defined. In the very low temperature limit $\left(T_{H} \ll \kappa^{2} \mu\right)$, the expectation value of the number operator is proportional to $\mu^{3}$. If there exists a Fermi surface, the Fermi surface energy leads to

$$
\epsilon_{F} \sim N^{1 / 3},
$$

which is similar to the RNAdS black brane case. Unlike the RNAdS black brane case, however, the trace of the boundary stress tensor shows a nonzero value depending on the chemical potential

$$
\left\langle T_{a}^{a}\right\rangle_{\mu}=E-3 P V=-\frac{16}{3} \kappa^{6} V_{3} \mu^{4},
$$

where the subscript $\mu$ implies a nontrivial vacuum state $|0\rangle_{\mu}$. This result indicates that the dual matter breaks the conformal symmetry. Why does not the trace of the boundary stress tensor vanish in spite of the asymptotic AdS geometry? The reason is as follows. Although the deformation of the asymptotic AdS geometry by the dilaton and gauge field does not change the leading behavior of the metric, it can contribute some subleading corrections to the metric at $r^{-2}$ order. Since these subleading terms provide nonzero finite contribution to both the on-shell action and boundary stress tensor even at the UV cutoff, the dual conformal symmetry is broken without changing the leading asymptotic geometry.

In order to describe the canonical ensemble instead of the grand canonical ensemble, we should impose the Neumann boundary condition on the vector field which requires one more boundary term

$$
S_{\text {can }}=S_{\text {grand }}+\frac{1}{g^{2}} \int_{\partial \mathscr{M}} d^{4} x A_{\mu} J^{\mu},
$$

where $A_{\mu}=\left\{-i A_{t}, 0,0,0\right\}$ and $J^{\mu}=\{i Q, 0,0,0\}$. Since the new additional boundary term cancels Neumannizing term, the vector field variation is well defined. In the dual theory point of view, inserting this boundary term corresponds to the Legendre transformation because the new boundary term reduces to

$$
\frac{1}{g^{2}} \int_{\partial \mathscr{M}} d^{4} x A_{\mu} J^{\mu}=\beta \mu N .
$$

Therefore, (59) becomes

$$
F=\Omega+\mu N,
$$

where $F \equiv S_{\text {can }} / \beta$ and $\Omega \equiv S_{\text {grand }} / \beta$ denote the free energy and grand potential, respectively. As mentioned before, it represents the Legendre transformation between the grand canonical ensemble and canonical ensemble. The onshell action and boundary energy-momentum tensor in the canonical ensemble yield the following free energy, internal energy and pressure:

$$
\begin{aligned}
& F=-\frac{\pi^{4} V_{3}}{2 \kappa^{2}} T_{H}^{4}+2 \pi^{2} \kappa^{2} V_{3} T_{H}^{2} \mu^{2}+\frac{14}{3} \kappa^{6} V_{3} \mu^{4}, \\
& E=\frac{3 \pi^{4} V_{3}}{2 \kappa^{2}} T_{H}^{4}+6 \pi^{2} \kappa^{2} V_{3} T_{H}^{2} \mu^{2}+\frac{14}{3} \kappa^{6} V_{3} \mu^{4}, \\
& P=\frac{\pi^{4}}{2 \kappa^{2}} T_{H}^{4}+2 \pi^{2} \kappa^{2} T_{H}^{2} \mu^{2}+\frac{10}{3} \kappa^{6} \mu^{4},
\end{aligned}
$$


where since the basic variables of the canonical ensemble are $T_{H}, N$, and $V_{3}$, the chemical potential is given by a function of those variables:

$$
\begin{gathered}
\mu=\left(43^{1 / 3} \pi^{2} \kappa^{6} T_{H}^{2}-2^{1 / 3}\right. \\
\times\left(\sqrt{96 \pi^{6} \kappa^{18} T_{H}^{6}+81 \kappa^{16}\left(N^{2} / V_{3}^{2}\right)}\right. \\
\left.\left.\quad-9 \kappa^{8} \frac{N}{V_{3}}\right)^{2 / 3}\right) \\
\times\left(26^{2 / 3} \kappa^{4}\right. \\
\times\left(\sqrt{96 \pi^{6} \kappa^{18} T_{H}^{6}+81 \kappa^{16}\left(N^{2} / V_{3}^{2}\right)}\right. \\
\left.\left.-9 \kappa^{8} \frac{N}{V_{3}}\right)^{1 / 3}\right)^{-1} .
\end{gathered}
$$

The holographic renormalization results in (62) coincide with the black brane thermodynamics and satisfies all thermodynamic relations.

\section{Discussion}

We have studied the holographic renormalization of the charged black branes whose asymptotic geometry is given by an AdS space. In the general renormalization scheme of the field theory, finding correct counter terms is very important because they cancel the UV divergences and at same time provide some finite contributions. Therefore, the renormalized physical quantities usually depend on the finite contributions of the counter terms. This is also true in the holographic renormalization. Following the AdS/CFT correspondence and using the holographic renormalization, in this paper, we have investigated the physical quantities of the dual field theory with matter. As mentioned before, for obtaining the correct physical results one should take into account the correct counter terms. In the charged black brane with an asymptotic AdS geometry, the bulk gauge field dual to matter does not require new divergent terms in the on-shell gravity action so that one need to introduce more counter terms except one used in the Schwarzschild (neutral) AdS black brane. Although there are no additional counter terms, the change of the metric caused by the bulk gauge field leads to a new finite contribution to the on-shell gravity action which depends on the physical quantities of matter, the chemical potential, or number density.

In the RNAdS black brane case, the boundary stress tensor generated by the on-shell gravity action becomes traceless. As expected usually, the dual matter does not break the conformal symmetry of the asymptotic AdS geometry. Furthermore, the expectation value of the number operator shows that the Fermi surface energy at zero temperature is proportional to $N^{1 / 3}$ which intriguingly resembles that of the free relativistic fermion. In spite of the resemblance, however, one can not identify the dual matter of the RNAdS black brane with a free relativistic fermion due to the absence of the asymptotic freedom. So it is more preferable to identify the dual matter with the non-Fermi liquid, strongly interacting fermion.

In the RNAdS black brane, there is no nontrivial dilaton field associated with the running coupling constant of the dual field theory. We further regarded the charged black brane in the Einstein-Maxwell-dilaton theory. The asymptotic geometry of it is also given by an AdS space, which is the leading part of the metric, and the next to leading part gives rise to the nontrivial finite contribution to both the onshell gravity action and the boundary stress tensor. Unlike the RNAdS black brane, the boundary stress tensor of the charged dilatonic black brane is not traceless which implies that the dual matter breaks the conformal symmetry although the leading part of the asymptotic metric is not changed.

Finally, we also showed that the holographic renormalization results can be reinterpreted as two different ways depending on the asymptotic boundary condition of the gauge field. These results are exactly matched with the thermodynamic laws of the charged black brane geometries that we considered. All above results are derived at the UV fixed point. So it is interesting to investigate the renormalizaiton group flow of them.

\section{Appendix}

\section{Thermodynamics of the RNAdS Black Brane}

Here, we will explain the thermodynamics of the RNAdS black brane in detail for the comparison with the holographic renormalization results. The action for the RNAdS black brane with a Lorentzian signature is given by

$$
S_{L}=\int d^{5} x \sqrt{-g}\left[\frac{1}{2 \kappa^{2}}(\mathscr{R}-2 \Lambda)-\frac{1}{4 g^{2}} F_{M N} F^{M N}\right],
$$

where $\Lambda=-12 / R^{2}$ and $R$ is the AdS radius. From now on, we set $R$ to be 1 for convenience. The Einstein and Maxwell equations lead to

$$
\begin{aligned}
& \mathscr{R}_{M N}-\frac{1}{2} g_{M N} \mathscr{R}+g_{M N} \Lambda \\
& =\frac{\kappa^{2}}{g^{2}}\left(F_{M P} F_{N}^{P}-\frac{1}{4} g_{M N} F_{P Q} F^{P Q}\right), \\
& 0=\partial_{M}\left(\sqrt{-g} g^{M P} g^{N Q} F_{P Q}\right) .
\end{aligned}
$$

If we turn only on the time component of the gauge field $A_{t}$ as a function of the radial coordinate $r$, the RNAdS black brane solution satisfying these two equations is given by

$$
\begin{gathered}
A_{t}=g^{2} \mu-\frac{Q}{2 r^{2}}, \\
d s^{2}=-r^{2} f(r) d t^{2}+\frac{1}{r^{2} f(r)} d r^{2}+r^{2} \delta_{i j} d x^{i} d x^{j},
\end{gathered}
$$


with

$$
f(r)=1-\frac{m}{r^{4}}+\frac{\kappa^{2}}{6 g^{2}} \frac{Q^{2}}{r^{6}},
$$

where $\mu$ and $Q$ are two arbitrary integration constants describing the electric chemical potential and charge density, respectively. Imposing an appropriate asymptotic boundary condition on $A_{t}$ can fix one of two integration constants. There are two possibilities which provide explicit thermodynamic meanings to constants. One is the Dirichlet boundary condition which determines the boundary value of $A_{t}, \mu$ and the other is the Neumann boundary condition in order to fix the charge $Q$.

Let us first take into account the case with the Neumann boundary condition. At the asymptotic boundary, the Neumann boundary condition for the gauge field is given by $Q$ :

$$
Q=\lim _{r \rightarrow \infty} \sqrt{-g} g^{r r} g^{t t} F_{r t},
$$

which fix the electric charge density as Q. Note that the chemical potential still remains as an undetermined integration constant. Due to the vanishing of $f(r)$ at the event (or outer) horizon $r_{h}$, the black brane mass density can be rewritten, in terms of $r_{h}$ and $Q$, as

$$
m=r_{h}^{4}+\frac{\kappa^{2}}{6 g^{2}} \frac{Q^{2}}{r_{h}^{2}} .
$$

Using this, the Hawking temperature defined by the surface gravity at the horizon reduces to

$$
T_{H}=\frac{1}{\pi} r_{h}-\frac{\kappa^{2} Q^{2}}{12 \pi g^{2}} \frac{1}{r_{h}^{5}} .
$$

It is well known that a black brane (or hole) provides a well-defined thermodynamic system, so one can regard, in the thermodynamic description, $r_{h}$ as a function of the other thermodynamic variables, $T_{H}$ and $Q$, from (A.8). The Bekenstein-Hawking entropy reads

$$
S_{\mathrm{BH}}=\frac{2 \pi V_{3}}{\kappa^{2}} r_{h}^{3},
$$

where $V_{3}$ is a regularized 3-dimensional spatial volume. Introducing the total charge $N=Q V_{3}$, the RNAdS black brane geometry can be classified by $T_{H}, N$, and $V_{3}$, which are thermodynamic variables describing the free energy of the canonical ensemble. From the thermodynamic relations together with (A.8) and (A.9), one can easily derive other thermodynamic quantities like the free energy $F$, internal energy $E$, and pressure $P$.

In the canonical ensemble, the most important thermodynamic function is the free energy

$$
F=E-T_{H} S_{\mathrm{BH}}
$$

from which all thermodynamic quantities can be derived. Applying the first law of thermodynamics,

$$
d E=T_{H} d S_{\mathrm{BH}}+\mu d N-P d V_{3},
$$

the free energy of the canonical ensemble satisfies the following thermodynamic relation:

$$
d F=-S_{\mathrm{BH}} d T_{H}+\mu d N-P d V_{3} .
$$

Since a RNAdS black brane solution in (A.4) is described by $Q=N / V_{3}$, it can be easily reinterpreted as thermodynamics of a canonical ensemble. After rewriting the Hawking temperature as a function of the Bekenstein-Hawking entropy with a fixed volume and total charge, the integral of the first thermodynamic law gives rise to

$$
E=\int d S_{\mathrm{BH}} T_{H}=\frac{3 V_{3}}{2 \kappa^{2}} r_{h}^{4}+\frac{N^{2}}{4 g^{2} V_{3}} \frac{1}{r_{h}^{2}} .
$$

In the above integration, it is also possible to add an arbitrary function depending only on $N$ and $V_{3}$ because including such a function still satisfies

$$
T_{H}=\left.\frac{\partial E}{\partial S_{\mathrm{BH}}}\right|_{N, V_{3}}
$$

However, this additional function does not satisfy the other thermodynamic relations so that such a term should vanish. Then, (A.10) gives rise to the free energy

$$
F=-\frac{V_{3}}{2 \kappa^{2}} r_{h}^{4}+\frac{5 N^{2}}{12 g^{2} V_{3}} \frac{1}{r_{h}^{2}},
$$

and when temperature and the total charge are fixed its derivative with respect to the volume leads to the pressure

$$
P=\left.\frac{\partial F}{\partial V_{3}}\right|_{T_{H}, N}=\frac{1}{2 \kappa^{2}} r_{h}^{4}+\frac{N^{2}}{12 g^{2} V_{3}^{2}} \frac{1}{r_{h}^{2}} .
$$

From the thermodynamic relation, the chemical potential of this system is given by a function of $N$ :

$$
\mu=\left.\frac{\partial F}{\partial N}\right|_{T_{H}, V_{3}}=\frac{N}{2 g^{2} V_{3}} \frac{1}{r_{h}^{2}},
$$

which is nothing but the regularity condition for the time component of the gauge field in (8). Note that $r_{h}$ in the canonical ensemble should be regarded as a function of $T_{H}$, $N$, and $V_{3}$. The above results show that the equation of state parameter of the dual system is given by $1 / 3$ like a pure $A d S_{5}$ and Schwarzschild-type AdS black brane. Therefore, the dual matter of the RNAdS black brane is conformal because the trace of the energy momentum tensor is zero; $E-3 P V_{3}=0$.

Now, let us consider the Dirichlet boundary condition instead of the Neumann boundary condition, which is given at the asymptotic boundary by

$$
\mu=\lim _{r \rightarrow \infty} \frac{A_{t}}{g^{2}} .
$$

In the thermodynamic interpretation, the chemical potential is a fundamental variable of the grand potential in the grand canonical ensemble. So imposing the Dirichlet boundary condition is associated with the description of the grand 
canonical ensemble. In this case, the grand potential is given by a function of temperature, chemical potential, and spatial volume, $\Omega\left(T_{H}, \mu, V_{3}\right)$. Moreover, the charge $N$ should be a function of $\mu$ and $T_{H}$, which can be derived from $N=$ $-\left.(\partial \Omega / \partial \mu)\right|_{T_{H}, V_{3}}$. In the above RNAdS black brane geometry, all solutions are not represented by $\mu$ but by $Q$. Therefore, one can not directly read the thermodynamics of the grand canonical ensemble without any additional relation. Using the regularity of the $A_{t}$ norm at the horizon, $Q$ can be fixed as a function of $\mu$ :

$$
Q=2 g^{2} r_{h}^{2} \mu
$$

which is related to the Legendre transformation. In the grand canonical ensemble description, $r_{h}$ must be regarded as a function of $T_{H}, \mu$, and $V_{3}$. Similar to the previous case, the first thermodynamic law and the following relations:

$$
\begin{gathered}
\Omega=E-T_{H} S_{\mathrm{BH}}-\mu N, \\
d \Omega=-S_{\mathrm{BH}} d T_{H}-N d \mu-P d V_{3},
\end{gathered}
$$

determine the grand potential as the following form:

$$
\Omega\left(T_{H}, \mu, V_{3}\right)=-\frac{V_{3}}{2 \kappa^{2}} r_{h}^{4}-\frac{g^{2} \mu^{2} V_{3}}{3} r_{h}^{2} .
$$

As mentioned before, the grand potential is related to the free energy by the Legendre transformation:

$$
\Omega=F-\mu N
$$

The other interesting thermodynamic quantities read

$$
\begin{aligned}
& E=\frac{3 V_{3}}{2 \kappa^{2}} r_{h}^{4}+g^{2} V_{3} \mu^{2} r_{h}^{2}, \\
& P=\frac{1}{2 \kappa^{2}} r_{h}^{4}+\frac{1}{3} g^{2} \mu^{2} r_{h}^{2},
\end{aligned}
$$

which are equivalent to the results obtained in the canonical ensemble if rewriting the chemical potential as a function of the charge by using (A.17).

\section{Conflict of Interests}

The author declares that there is no conflict of interests regarding the publication of this paper.

\section{Acknowledgments}

The author would like to thank Yun Soo Myung and $\mathrm{Mu}-$ in Park for valuable discussion. This work has been supported by the WCU Grant no. R32-10130 and the Research Fund no. 1-2008-2935-001-2 by Ewha Womans University. The author was also supported by Basic Science Research Program through the National Research Foundation of Korea (NRF) funded by the Ministry of Education (Grant no. NRF2013R1A1A2A10057490).

\section{References}

[1] J. Maldacena, "The large $N$ limit of superconformal field theories and supergravity," Advances in Theoretical and Mathematical Physics, vol. 2, no. 2, pp. 231-252, 1998.

[2] S. S. Gubser, I. R. Klebanov, and A. M. Polyakov, "Gauge theory correlators from non-critical string theory," Physics Letters B, vol. 428, no. 1-2, pp. 105-114, 1998.

[3] E. Witten, "Anti de Sitter space and holography," Advances in Theoretical and Mathematical Physics, vol. 2, no. 2, pp. 253-291, 1998.

[4] E. Witten, "Anti-de Sitter space, thermal phase transition, and confinement in gauge theories," Advances in Theoretical and Mathematical Physics, vol. 2, no. 3, pp. 505-532, 1998.

[5] V. Balasubramanian and P. Kraus, "Spacetime and the holographic renormalization group," Physical Review Letters, vol. 83, no. 18, pp. 3605-3608, 1999.

[6] V. Pestun, "Localization of the four-dimensional N $=4$ SYM to a two-sphere and 1/8 BPS Wilson loops," Journal of High Energy Physics, vol. 2012, article 067, 2012.

[7] J. A. Minahan and K. Zarembo, "The Bethe-ansatz for Script $\mathcal{N}=4$ super Yang-Mills," Journal of High Energy Physics, vol. 2003, no. 03, article 013, 2003.

[8] N. Beisert and M. Staudacher, "The $N=4$ SYM integrable super spin chain," Nuclear Physics B, vol. 670, no. 3, pp. 439-463, 2003.

[9] B.-H. Lee, C. Park, and S.-J. Sin, "A dual geometry of the hadron in dense matter," Journal of High Energy Physics, vol. 2009, no. 07, article 087, 2009.

[10] C. Park, "Dissociation of a heavy meson in the quark medium," Physical Review D, vol. 81, Article ID 045009, 2010.

[11] K. Jo, B.-H. Lee, C. Park, and S.-J. Sin, "Holographic QCD in medium: a bottom up approach," Journal of High Energy Physics, vol. 2010, no. 6 , article 22, 2010.

[12] J. D. Brown and J. W. York Jr., "Quasilocal energy and conserved charges derived from the gravitational action," Physical Review $D$, vol. 47, p. 1407, 1993.

[13] V. Balasubramanian and P. Kraus, "A stress tensor for anti-de Sitter gravity," Communications in Mathematical Physics, vol. 208, no. 2, pp. 413-428, 1999.

[14] M. Henningson and K. Skenderis, "The holographic Weyl anomaly," Journal of High Energy Physics, vol. 1998, article 023, 1998.

[15] M. Henningson and K. Skenderis, "Holography and the Weyl anomaly," Fortschritte der Physik, vol. 48, pp. 125-128, 2000.

[16] E. T. Akhmedov, "A remark on the AdS/CFT correspondence and the renormalization group flow," Physics Letters B, vol. 442, no. 1-4, pp. 152-158, 1998.

[17] S. de Haro, K. Skenderis, and S. N. Solodukhin, "Holographic reconstruction of spacetime and renormalization in the AdS/CFT correspondence," Communications in Mathematical Physics, vol. 217, no. 3, pp. 595-622, 2001.

[18] M. Bianchi, D. Z. Freedman, and K. Skenderis, "How to go with an RG flow," Journal of High Energy Physics, vol. 108, Article ID article 041, 2001.

[19] M. Bianchi, D. Z. Freedman, and K. Skenderis, "Holographic renormalization," Nuclear Physics B, vol. 631, no. 1-2, pp. 159194, 2002.

[20] K. Skenderis, "Lecture notes on holographic renormalization," Classical and Quantum Gravity, vol. 19, no. 22, pp. 5849-5876, 2002. 
[21] I. Papadimitriou and K. Skenderis, "Thermodynamics of asymptotically locally AdS spacetimes," Journal of High Energy Physics, vol. 2008, no. 8, article 004, 2005.

[22] E. T. Akhmedov, I. B. Gahramanov, and E. T. Musaev, "Hints on integrability in the Wilsonian/holographic renormalization group," JETP Letters, vol. 93, no. 9, pp. 545-550, 2011.

[23] K. Balasubramanian and J. McGreevy, "An analytic Lifshitz black hole," Physical Review D: Particles, Fields, Gravitation, and Cosmology, vol. 80, no. 10, Article ID 104039, 8 pages, 2009.

[24] Y. Korovin, K. Skenderis, and M. Taylor, "Lifshitz from AdS at finite temperature and top down models," Journal of High Energy Physics, vol. 2013, article 127, 2013.

[25] S. F. Ross and O. Saremi, "Holographic stress tensor for nonrelativistic theories," Journal of High Energy Physics, vol. 2009, article 009, 2009.

[26] Y. Korovin, K. Skenderis, and M. Taylor, "Lifshitz as a deformation of anti-de Sitter," Journal of High Energy Physics, vol. 2013, 2013.

[27] C. Park, "Notes on the holographic Lifshitz theory," Advances in High Energy Physics, vol. 2014, Article ID 917632, 9 pages, 2014.

[28] C. Park, "Massive quasinormal mode in the holographic Lifshitz theory," Physical Review D, vol. 89, Article ID 066003, 2014.

[29] K. Goldstein, S. Kachru, S. Prakash, and S. P. Trivedi, "Holography of charged dilaton black holes," Journal of High Energy Physics, vol. 1008, article 078, 2010.

[30] C. Charmousis, B. Gouteraux, B. S. Kim, E. Kiritsis, and R. Meyer, "Effective holographic theories for low-temperature condensed matter systems," Journal of High Energy Physics, vol. 2010, no. 11, article 151, 2010.

[31] M. Cadoni, S. Mignemi, and M. Serra, "Exact solutions with AdS asymptotics of Einstein and Einstein-Maxwell gravity minimally coupled to a scalar field," Physical Review D, vol. 84, Article ID 084046, 2011.

[32] K. Goldstein, N. Iizuka, S. Kachru, S. Prakash, S. P. Trivedi, and A. Westphal, "Holography of dyonic dilaton black branes," Journal of High Energy Physics, vol. 2010, article 27, 2010.

[33] X. Dong, S. Harrison, S. Kachru, G. Torroba, and H. Wang, "Aspects of holography for theories with hyperscaling violation," Journal of High Energy Physics, vol. 2012, article 41, 2012.

[34] L. Huijse, S. Sachdev, and B. Swingle, "Hidden Fermi surfaces in compressible states of gauge-gravity duality," Physical Review $B$, vol. 85, Article ID 035121, 2012.

[35] I. Kanitscheider and K. Skenderis, "Universal hydrodynamics of non-conformal branes," Journal of High Energy Physics, vol. 2009, article 062, 2009.

[36] B. Gouteraux, J. Smolic, M. Smolic, K. Skenderis, and M. Taylor, "Holography for Einstein-Maxwell-dilaton theories from generalized dimensional reduction," Journal of High Energy Physics, vol. 2012, article 089, 2012.

[37] S. Kulkarni, B.-H. Lee, C. Park, and R. Roychowdhury, "Nonconformal hydrodynamics in Einstein-dilaton theory," Journal of High Energy Physics, vol. 2012, article 4, 2012.

[38] C. Park, "Holographic aspects of a relativistic nonconformal theory," Advances in High Energy Physics, vol. 2013, Article ID 389541, 16 pages, 2013.

[39] S. Kulkarni, B.-H. Lee, J.-H. Oh, C. Park, and R. Roychowdhury, "Transport in non-conformal holographic fluids," Journal of High Energy Physics, vol. 2013, article 149, 2013.

[40] C. Park, "Holographic symmetry energy of the nuclear matter," Physics Letters B, vol. 708, no. 3-5, pp. 324-329, 2012.
[41] B.-H. Lee, S. Mamedov, S. Nam, and C. Park, "Holographic meson mass splitting in the Nuclear Matter," Journal of High Energy Physics, vol. 1308, no. 045, 2013.

[42] A. Chamblin, R. Emparan, C. V. Johnson, and R. C. Myers, "Holography, thermodynamics, and fluctuations of charged AdS black holes," Physical Review D: Third Series, vol. 60, no. 10, Article ID 104026, 1999.

[43] R. Emparan, C. V. Johnson, and R. C. Myers, "Surface terms as counterterms in the AdS-CFT correspondence," Physical Review D, vol. 60, no. 10, Article ID 104001, 14 pages, 1999.

[44] H. Liu, J. McGreevy, and D. Vegh, "Non-Fermi liquids from holography," Physical Review D, vol. 83, Article ID 065029, 2011.

[45] S. S. Gubser and F. D. Rocha, "Peculiar properties of a charged dilatonic black hole in AdS_5," Physical Review D, vol. 81, Article ID 046001, 2010.

[46] R. A. Davison, M. Goykhman, and A. Parnachev, "AdS/CFT and Landau Fermi liquids," Journal of High Energy Physics, vol. 1407, no. 109, 2014.

[47] S. S. Gubser and J. Ren, "Analytic fermionic Green's functions from holography," Physical Review D, vol. 86, Article ID 046004, 2012.

[48] M. Alishahiha, M. R. M. Mozaffar, and A. Mollabashi, "Holographic aspects of two-charged dilatonic black hole in $\mathrm{AdS}_{5}$," Journal of High Energy Physics, vol. 2013, article 3, 2012. 

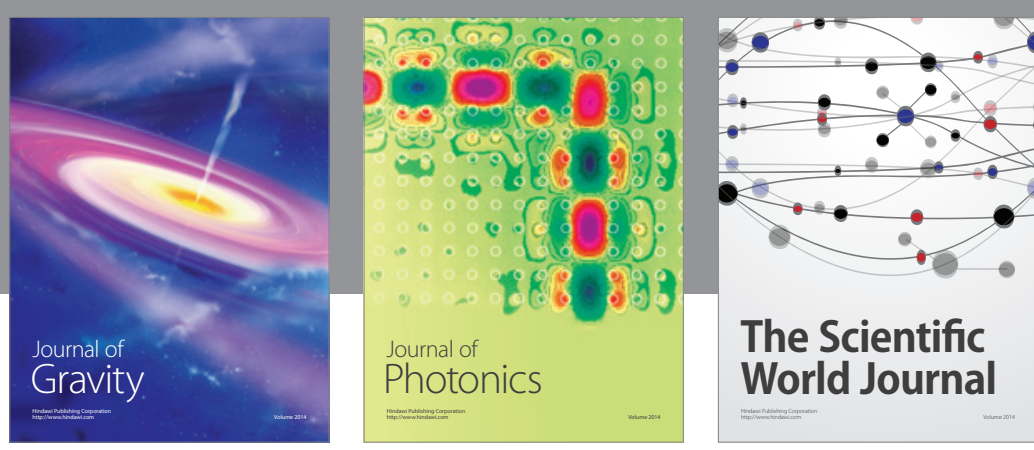

The Scientific World Journal
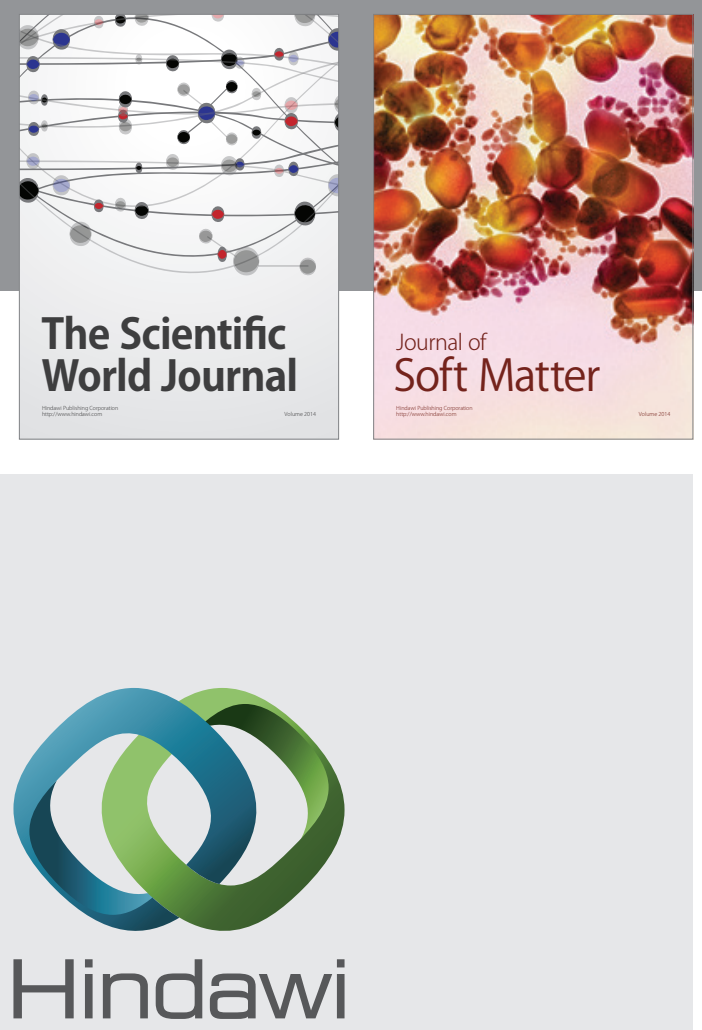

Submit your manuscripts at

http://www.hindawi.com

nternational Journal of

Statistical Mechanics
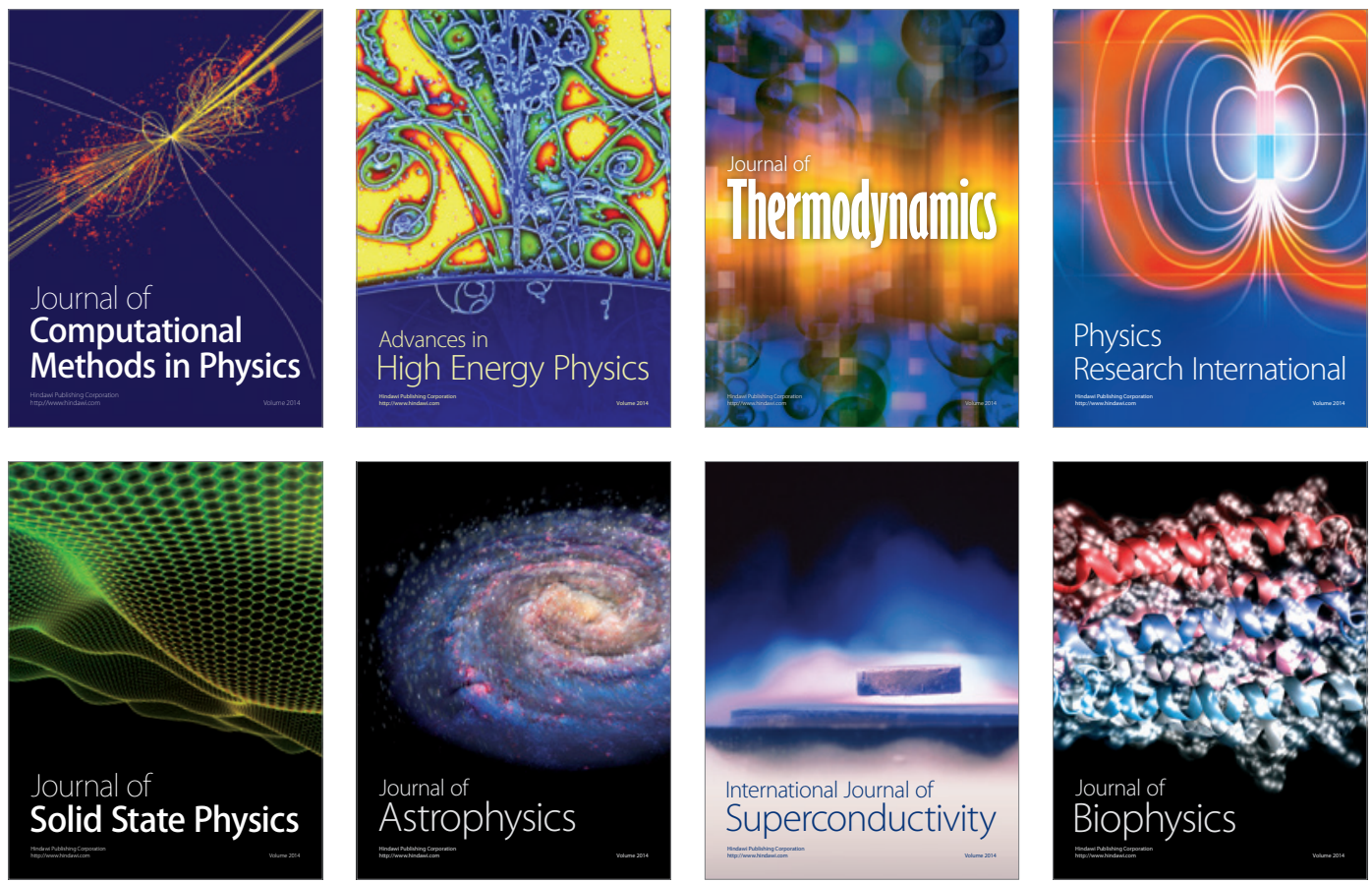
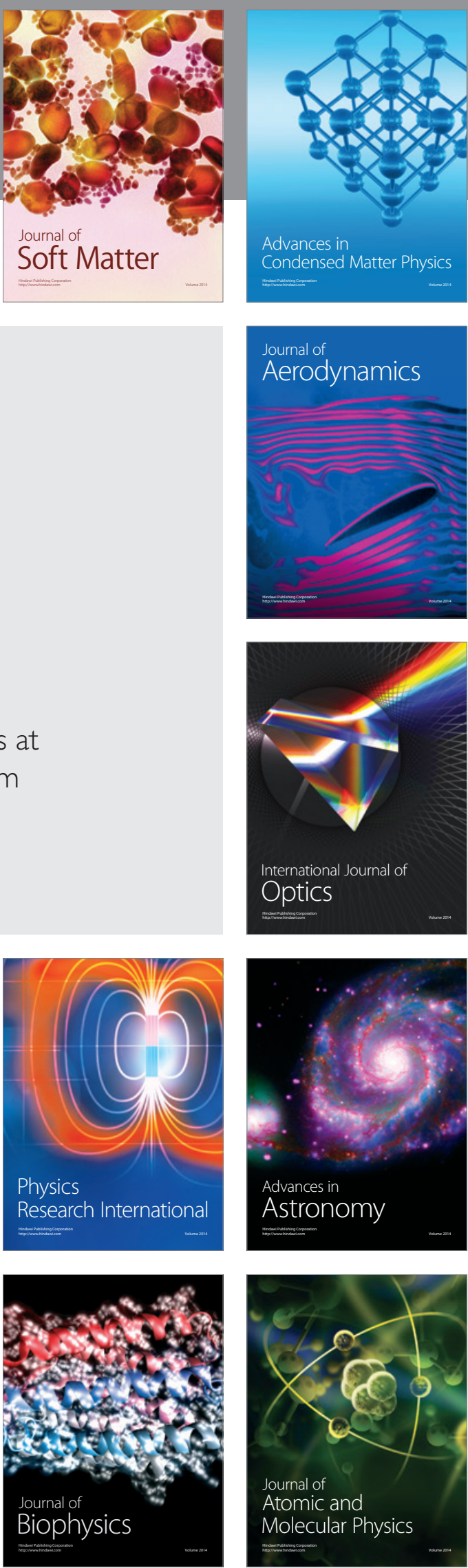